\title{
Spherical Indentation of a Freestanding Circular Membrane Revisited: Analytical Solutions and Experiments
}

\author{
Congrui Jin ${ }^{\mathrm{a}, *}$, Ali Davoodabadi ${ }^{\mathrm{a}}$, Jianlin Li ${ }^{\mathrm{b}}$, Yanli Wang ${ }^{\mathrm{c}}$, Timothy \\ Singler ${ }^{a}$ \\ ${ }^{a}$ Department of Mechanical Engineering, State University of New York at Binghamton, \\ Vestal, NY 13850, USA \\ ${ }^{b}$ Energy and Transportation Science Division, Oak Ridge National Laboratory, Oak \\ Ridge, TN 37831, USA \\ ${ }^{c}$ Materials Science and Technology Division, Oak Ridge National Laboratory, Oak Ridge, \\ TN 37831, USA
}

\begin{abstract}
Due to the development of novel micro-fabrication techniques to produce ultra-thin materials and increasing interest in thin biological membranes, in recent years, the mechanical characterization of thin films has received a significant amount of attention. To provide a more accurate solution to predict the relationship among contact radius, load and deflection, the fundamental and widely applicable problem of spherical indentation of a freestanding circular membrane has been revisited. The work presented here significantly extends the previous contributions by providing an exact analytical solution to the governing equations of Föppl-Hecky membrane indented by a frictionless spherical indenter. In this study, experiments of spherical indentation has been performed, and the exact analytical solution presented in this paper is compared against experimental data from existing literature as well as our own experimental results.
\end{abstract}

Keywords: Membrane, Thin Film, Spherical Indentation, Analytical Solution, Experiments

\footnotetext{
${ }^{*}$ Corresponding Author

Email address: cjin@binghamton.edu (Congrui Jin)
} 


\section{Introduction}

Mechanical testing of freestanding films offers many advantages over other methods, as it avoids the complicating role of the substrate whose properties strongly affect the test for thin films, circumvents complicating factors associated with surface conditions and the film-substrate interface, and leads to simpler stress distributions that are more easily connected to measured forces and displacements. Some examples of experiments on freestanding films include the uniaxial tensile test (Haque and Saif, 2002; Ben-David et al., 2014), the bulge test (Small and Nix, 1990; Vlassak and Nix, 1992; Poilane et al., 2000; Zheng et al., 2000), and a membrane deflection technique (Espinosa and Prorok, 2001; Espinosa et al., 2003). While attractive in many respects, these experimental methods require nearly defect-free films without pinholes or porosity, and as such is not feasible for many material systems, such as polymers, biological membranes, and porous low- $k$ dielectrics. Indentation testing of freestanding films is increasingly feasible with the growing availability of increasingly sophisticated nano-mechanical testing systems (Liu and Ju, 2001; Ju et al., 2002, 2004; Scott et al., 2004; Leseman and Mackin, 2007). The mechanics of spherical indentation of freestanding circular thin films has been previously studied by Begley and Mackin (2004), and since then, their results have been widely cited as a standard to extract mechanical properties from indentation testing of freestanding films (Scott et al., 2004; Leseman and Mackin, 2007; Chua and Oyen, 2009; Rausch and Kuhl, 2013; Kang et al., 2013).

A schematic diagram of the geometry under consideration is shown in Fig. 1. In a typical setup, an elastic membrane with an elastic modulus $E$, Poisson's ratio $\nu$, thickness $h$, and radius $a$ is clamped at the perimeter before indentation. A frictionless spherical indenter of radius $R$ is brought into contact with the membrane by applying a transverse load $P_{0}$ at the center of the membrane. The maximum transversal displacement of the noncontact region is denoted by $w_{0}$, the centerline displacement of membrane is denoted by $d$, and the corresponding contact radius is denoted by $c$. To limit indentation stress and avoid highly localized deformation, such as cracking and yielding, the indenter radius must be a significant fraction of the freestanding span, which implies that the point-load solutions, such as the well-known Schwerin solution (Schwerin, 1929), must be replaced by analyses that include explicit treatment of the contact region.

The most widely cited results from the article by Begley and Mackin 
(2004) are the approximate closed-form solutions as summarized below. The closed-form solutions are obtained by adopting the classic Föppl-Hecky small finite-deflection theory (Föppl, 1907; Hencky, 1915; Bhatia and Nachbar, 1968; Jin and Wang, 2008) and developing two different approximations: one for the contact zone and one for the freestanding region. For the contact region approximation, it has been assumed that stretch ratios are approximately equal to the average radial stretch. To describe the response of the freestanding region, it has been assumed that circumferential strains are negligible in comparison to radial strains. The approximate solutions in each region are related through the indenter load, which is used to determine integration constants at the edge of each region. According to the approximate solutions, the contact radius as a function of applied load is given by

$$
\frac{c}{R}=\sqrt{\sqrt{\left(\frac{3}{2 \pi}\right)\left(\frac{P_{0}}{E h R}+6 \pi \epsilon_{0}^{2}\right)}-3 \epsilon_{0}}
$$

where the prestrain $\epsilon_{0}$ is related to the prestress used in this paper by $\epsilon_{0}=$ $(1-\nu) N_{0} / E h$. Inside the contact region, the indentation strain is given by

$$
\epsilon_{m}=\epsilon(r=0)-\epsilon_{0}=\frac{1}{6} \sqrt{\left(\frac{3}{2 \pi}\right)\left(\frac{P_{0}}{E h R}+6 \pi \epsilon_{0}^{2}\right)}-\frac{1}{2} \epsilon_{0}
$$

The load-deflection response of the membrane is given by

$$
\frac{d}{R}=\frac{2}{3 \pi}\left(\frac{a}{R}\right)^{3 / 4} \frac{\left[\sqrt{9 \pi^{4}\left(P_{0} / E h R\right)^{2}+64 \pi^{6} \epsilon_{0}^{3}}+3 \pi^{2}\left(P_{0} / E h R\right)\right]^{2 / 3}-4 \pi^{3} \epsilon_{0}}{\left[\sqrt{9 \pi^{4}\left(P_{0} / E h R\right)^{2}+64 \pi^{6} \epsilon_{0}^{3}}+3 \pi^{2}\left(P_{0} / E h R\right)\right]^{1 / 3}}
$$

In the limit of zero prestrain, Eq. (3) simplifies to

$$
\frac{d}{R}=\left(\frac{a}{R}\right)^{3 / 4}\left(\frac{16}{9 \pi} \frac{P_{0}}{E h R}\right)^{1 / 3}
$$

Clearly, the approximate solution does not satisfy, simultaneously, both equilibrium equations and continuity of displacements, and hence is not asymptotically exact. When the indenter radius is much smaller than that of the circular membrane, it is reasonable to assume the applied force as a point load, however, the approximate solutions provided by Begley and Mackin (2004) do not converge to the Schwerin-type point-load solution (Schwerin, 1929; Komaragiri et al., 2005; Jin and Wang, 2008) as $R / a \rightarrow 0$. In fact, 
the approximate solution predicts an infinite deflection under a central point load. Moreover, in such spherical indentation experiments, either radial stress (Bhatia and Nachbar, 1968) or radial displacement (Scott et al., 2004) can be prescribed at the boundary of the circular film, but the approximate solution does not consider the effect of different boundary conditions.

It is important to note that the first study to explicitly include treatment of the finite contact region during indentation of membranes was provided by Bhatia and Nachbar (1968). Föppl-Hecky theory was applied to study the mechanics of spherical indentation, but as illustrated by these authors, rigorous asymptotic solutions require that the integration constants be determined by matching the solution inside the contact region to the solution in the freestanding region, which is a fairly complicated procedure that only yields closed-form results in two limiting scenarios: the "small loads" scenario, which yields a simple linear expression for load-deflection behavior; and the "large loads" scenario, which yields nonlinear load-deflection behavior and requires numerical solutions.

The work presented here significantly extends the previous contributions by providing an exact analytical solution to the governing equations of FöpplHecky membrane indented by a frictionless spherical indenter. The exact solution does not have the limitations of the approximate solution. In our analysis, at the membrane boundary, we allow either radial stress or radial displacement to be prescribed. To verify our analytical exact solutions, experiments are performed and a comparison of existing solutions with experimental results is provided.

\section{Governing Equations of Föppl-Hecky Membrane}

The classic Föppl-Hencky membrane theory is derived based on the assumptions that the strains are sufficiently small so that linear stress-strain relations are approximately valid and that the magnitude of rotations is sufficiently restricted (Föppl, 1907; Hencky, 1915; Bhatia and Nachbar, 1968). In the plane of the circular membrane, there are the actions of the radial membrane force $N_{r}$ and the circumferential membrane force $N_{\theta}$. The in-plane equilibrium equation gives

$$
N_{r}-N_{\theta}+r \frac{d N_{r}}{d r}=0
$$

Assume that the radial strain is $\epsilon_{r}$, the circumferential strain is $\epsilon_{\theta}$, the radial displacement is $u$, and the transversal displacement is $w$. The relationship 
of the strain and the displacement for a large-deflection membrane can be written as:

$$
\begin{gathered}
\epsilon_{r}=\frac{d u}{d r}+\frac{1}{2}\left(\frac{d w}{d r}\right)^{2} \\
\epsilon_{\theta}=\frac{u}{r}
\end{gathered}
$$

Substituting (7) into (6) gives,

$$
r \frac{d \epsilon_{\theta}}{d r}+\epsilon_{\theta}-\epsilon_{r}+\frac{1}{2}\left(\frac{d w}{d r}\right)^{2}=0
$$

The material is assumed to follow Hooke's law, i.e. for a material with Young's modulus $E$ and Poisson's ratio $\nu$, we have

$$
N_{r}=\frac{E h}{1-\nu^{2}}\left(\epsilon_{r}+\nu \epsilon_{\theta}\right) \quad N_{\theta}=\frac{E h}{1-\nu^{2}}\left(\epsilon_{\theta}+\nu \epsilon_{r}\right)
$$

The out-of-plane equilibrium condition gives

$$
\frac{d}{d r}\left(r N_{r} \frac{d w}{d r}\right)+r q=0
$$

where $q$ is the force density. Integrating both sides once with respect to $r$ gives

$$
N_{r} \frac{d w}{d r}=-\psi
$$

where

$$
\psi=\frac{1}{r} \int_{0}^{r} s q(s) \mathrm{d} s
$$

\subsection{Free region of membrane}

The noncontact overhanging part $(c \leq r \leq a)$ is not subject to any load. Therefore, the central point load applied to the indenter $q(r)=P_{0} \delta(r)$ is distributed to a line at the contact edge $r=c$, where $\delta(r)$ is the Dirac delta function, and thus $\psi=P_{0} / 2 \pi r$. Substituting Eq. (9) into Eq. (8) and making use of Eqs. (5) and (11), we obtain the following governing equations of the Föppl-Hencky membrane (Jin and Wang, 2008):

$$
\begin{gathered}
r \frac{d}{d r}\left[\frac{d}{r d r}\left(r^{2} N_{r}\right)\right]=-\frac{h E}{2}\left(\frac{d w}{d r}\right)^{2} \\
N_{r} \frac{d w}{d r}=-\frac{P_{0}}{2 \pi r}
\end{gathered}
$$


In the presence of residual stress, we allow either radial stress or radial displacement to be prescribed: (a) $w=0, N_{r}=N_{0}$ at $r=a$; and (b) $w=0, u=u_{0}$ at $r=a$. $N_{0}$ is positive for pre-tension and negative for pre-compression. It is interesting to note that in the study provided by Bhatia and Nachbar (1968) the circular membrane is pre-stretched in its plane by dead-weight loading, corresponding to boundary condition (a), while in the experiments performed by Scott et al. (2004) the radial displacement of the circular membrane is fixed at the boundary, corresponding to boundary condition (b). During the derivation of the approximate solution (Begley and Mackin, 2004), boundary condition (b) is always assumed and boundary condition (a) is never considered.

\subsection{Membrane in frictionless contact with indenter}

At the contact edge $r=c$, the condition of equilibrium of the indenter is used to obtain the following:

$$
\begin{gathered}
\left(N_{r}\right)_{c}=\frac{P_{0} R}{2 \pi c^{2}} \\
u_{c}=\frac{(1-\nu) P_{0} R}{2 \pi E h c}-\frac{c^{3}}{8 R^{2}}
\end{gathered}
$$

Following the derivation provided by Bhatia and Nachbar (1968), the stress and displacement based on Föppl-Hencky theory can be obtained as

$$
\begin{gathered}
\frac{N_{r}}{E h}=\frac{\left(N_{r}\right)_{c}}{E h}+\frac{1}{16}\left(\frac{c^{2}}{R^{2}}-\frac{r^{2}}{R^{2}}\right) \\
\frac{N_{\theta}}{E h}=\frac{\left(N_{r}\right)_{c}}{E h}+\frac{1}{16} \frac{c^{2}}{R^{2}}-\frac{3}{16} \frac{r^{2}}{R^{2}} \\
\frac{u}{r}=(1-\nu) \frac{\left(N_{r}\right)_{c}}{E h}+\frac{1-\nu}{16} \frac{c^{2}}{R^{2}}-\frac{3-\nu}{16} \frac{r^{2}}{R^{2}} \\
w=w_{0}+\frac{R}{2}\left(\frac{c^{2}}{R^{2}}-\frac{r^{2}}{R^{2}}\right)
\end{gathered}
$$

Based on Eqs. (19) and (20) we can obtain the maximum indentation strain, $\epsilon_{m}$, and centerline displacement of the membrane, $d$, respectively, as

$$
\begin{gathered}
\epsilon_{m}=\epsilon(r=0)=(1-\nu) \frac{\left(N_{r}\right)_{c}}{E h}+\frac{1-\nu}{16} \frac{c^{2}}{R^{2}} \\
d=w_{0}+\frac{c^{2}}{2 R}
\end{gathered}
$$




\section{Mathematical Derivation of the Exact Solution}

Introducing the dimensionless variables listed in Table 1, we can obtain the dimensionless forms of Eqs. (13) and (14) as

$$
\begin{gathered}
\frac{d^{2} S}{d y^{2}}=-\frac{\phi^{2}}{y^{2}} \\
S \phi+F y=0
\end{gathered}
$$

Substituting Eq. (23) into (24), we obtain

$$
\frac{d^{2} S}{d y^{2}}+\frac{F^{2}}{S^{2}}=0
$$

Stress and displacement in the freestanding region are given in terms of $S$ by

$$
\begin{gathered}
\frac{u}{r}=\frac{h^{2}}{8 a^{2}}\left[2 \frac{d S}{d y}-(1+\nu) \frac{S}{y}\right] \\
\frac{N_{r}}{E h}=\frac{h^{2}}{8 a^{2}} \frac{S}{y} \\
\frac{N_{\theta}}{E h}=\frac{h^{2}}{8 a^{2}}\left(2 \frac{d S}{d y}-\frac{S}{y}\right)
\end{gathered}
$$

The two boundary conditions become

$$
\begin{gathered}
\text { (a) } \quad S=n_{0} \quad \text { at } y=1 \\
\text { (b) } 2 \frac{d S}{d y}-(1+\nu) S=(1-\nu) n_{0} \quad \text { at } y=1
\end{gathered}
$$

At the contact edge $y=\zeta$, continuity conditions of displacement and normal stress give the following equations:

$$
\begin{gathered}
S=\lambda F \\
\frac{d S}{d y}=\frac{\lambda F}{\zeta}-\frac{\zeta}{2 \lambda^{2}}
\end{gathered}
$$

It is well known that the conditions $N_{r} \geq 0$ and $N_{\theta} \geq 0$ need to hold everywhere in the Föppl-Hencky membrane for the equilibrium configuration to be physically admissible (Steigmann, 1986; Komaragiri et al., 2005; Jin 
and Wang, 2008). Negative stress in any part of the membrane may open the possibility of wrinkling. Since $N_{r} \geq 0$ everywhere, the integral of Eq. (25) has the following form

$$
\frac{d S}{d y}=\left[\frac{2\left(F^{2}-C_{1} S\right)}{S}\right]^{1 / 2}
$$

which will be considered in the following three cases depending on the integration constant $C_{1}$.

- Branch 1: $C_{1}>0$

We here introduce a variable $\theta$ such that

$$
S=\frac{F^{2}}{C_{1}} \sin ^{2} \theta
$$

where $0<\theta<\pi / 2$. Substituting Eq. (34) into Eq. (33) we obtain

$$
\frac{d \theta}{d y}=2^{-1 / 2} C_{1}^{3 / 2} F^{-2} \sin ^{-2} \theta
$$

Integrating Eq. (35) gives

$$
y+C_{2}=\left(2 C_{1}\right)^{-3 / 2} F^{2}(2 \theta-\sin 2 \theta)
$$

where $C_{2}$ is another integration constant. Denoting $\theta(y=1)=\theta_{m}$ and $\theta(y=\zeta)=\theta_{n}$ and substituting $y=1$ and $y=\zeta$ into Eq. (36) gives

$$
1-\zeta=\left(2 C_{1}\right)^{-3 / 2} F^{2}\left(2 \theta_{m}-\sin 2 \theta_{m}-2 \theta_{n}+\sin 2 \theta_{n}\right)
$$

Eqs. (31) and (32) are then transformed into the following equations:

$$
\begin{gathered}
\frac{F}{C_{1}} \sin ^{2} \theta_{n}=\lambda \\
\left(2 C_{1}\right)^{1 / 2} \frac{\cos \theta_{n}}{\sin \theta_{n}}=\frac{\lambda F}{\zeta}-\frac{\zeta}{2 \lambda^{2}}
\end{gathered}
$$

Substituting Eq. (38) into Eq. (39) to delete $C_{1}$ gives

$$
\zeta=\left[\left(\cos ^{2} \theta_{n}+1\right)^{1 / 2}-\cos \theta_{n}\right]\left(2 F \lambda^{3}\right)^{1 / 2}
$$


The boundary conditions at $y=1$ then becomes:

$$
\begin{gathered}
\text { (a) } \frac{F^{2}}{C_{1}} \sin ^{2} \theta_{m}=n_{0} \\
\text { (b) } \frac{\left(2 C_{1}\right)^{3 / 2}}{F^{2}}=(1+\nu) \frac{\sin ^{3} \theta_{m}}{\cos \theta_{m}}+\frac{C_{1} n_{0}(1-\nu)}{F^{2}} \frac{\sin \theta_{m}}{\cos \theta_{m}}
\end{gathered}
$$

Substituting Eq. (38) into Eqs. (41) and (42) gives

$$
\text { (a) } \frac{\sin ^{2} \theta_{m}}{\sin ^{2} \theta_{n}}=\frac{n_{0}}{\lambda F}
$$

$$
\text { (b) }\left(\frac{8}{F \lambda^{3}}\right)^{1 / 2} \sin ^{3} \theta_{n}=(1+\nu) \frac{\sin ^{3} \theta_{m}}{\cos \theta_{m}}+\frac{n_{0}(1-\nu)}{\lambda F} \frac{\sin ^{2} \theta_{n} \sin \theta_{m}}{\cos \theta_{m}}
$$

Substituting Eqs. (38)-(40) into Eq. (37) gives

$$
\left(\cos ^{2} \theta_{n}+1\right)^{1 / 2}-\cos \theta_{n}+\frac{2 \theta_{m}-\sin 2 \theta_{m}-2 \theta_{n}+\sin 2 \theta_{n}}{4 \sin ^{3} \theta_{n}}=\left(2 F \lambda^{3}\right)^{-1 / 2}
$$

Substituting Eq. (34) into Eq. (24) and making use of Eq. (35) give

$$
\frac{w_{0}}{h}=\left(2 C_{1}\right)^{-1 / 2} F\left(\theta_{m}-\theta_{n}\right)
$$

Based on Eq. (22) we can obtain

$$
\frac{d}{h}=\frac{w_{0}}{h}+\frac{\zeta}{2 \lambda}
$$

By solving Eqs. (43)-(45), $\theta_{n}$ and $\theta_{m}$ can be obtained. It can then be used to determine $w_{0}$ and $d$ by making use of Eqs. (46) and (47). The conditions $N_{r} \geq 0$ and $N_{\theta} \geq 0$ need to hold everywhere in $\zeta<y<1$. Based on Eq. (34), we can obtain

$$
\begin{gathered}
\frac{8 a^{2} N_{r}}{E h^{3}}=\frac{F^{2}}{C_{1}} \frac{\sin ^{2} \theta}{y} \\
\frac{8 a^{2} N_{\theta}}{E h^{3}}=\frac{F^{2}}{C_{1}}\left[\frac{\left(2 C_{1}\right)^{3 / 2}}{F^{2}} \frac{\cos \theta}{\sin \theta}-\frac{\sin ^{2} \theta}{y}\right]
\end{gathered}
$$

To ensure that $N_{\theta} \geq 0$ we substitute Eq. (36) into Eq. (49) and obtain the following relationship:

$$
-\zeta \leq C_{2} \leq \zeta\left[\frac{\cos \theta_{n}}{\sin ^{3} \theta_{n}}\left(2 \theta_{n}-\sin 2 \theta_{n}\right)-1\right]
$$


- Branch 2: $C_{1}<0$

Denote $C_{1}=-\bar{C}_{1}$ where $\bar{C}_{1}$ is positive and introduce a variable $\theta$ such that

$$
S=\frac{F^{2}}{\bar{C}_{1}} \cot ^{2} \theta
$$

where $0<\theta<\pi / 2$. Substituting Eq. (51) into Eq. (33) we obtain

$$
\frac{d \theta}{d y}=-2^{-1 / 2} \bar{C}_{1}^{3 / 2} F^{-2} \sin ^{3} \theta \cos ^{-2} \theta
$$

Integrating Eq. (52), we obtain

$$
y+C_{2}=2^{-1 / 2} \bar{C}_{1}^{-3 / 2} F^{2}\left[\frac{\cos \theta}{\sin ^{2} \theta}-\ln \left(\cot \frac{\theta}{2}\right)\right]
$$

where $C_{2}$ is another integration constant. Substituting $y=1$ and $y=\zeta$ into Eq. (53) gives

$$
1-\zeta=2^{-1 / 2} \bar{C}_{1}^{-3 / 2} F^{2}\left[\frac{\cos \theta_{m}}{\sin ^{2} \theta_{m}}-\ln \left(\cot \frac{\theta_{m}}{2}\right)-\frac{\cos \theta_{n}}{\sin ^{2} \theta_{n}}+\ln \left(\cot \frac{\theta_{n}}{2}\right)\right]
$$

Eqs. (31) and (32) can then be transformed into the following equations:

$$
\begin{gathered}
\frac{F}{\bar{C}_{1}} \cot ^{2} \theta_{n}=\lambda \\
\left(2 \bar{C}_{1}\right)^{\frac{1}{2}} \frac{1}{\cos \theta_{n}}=\frac{\lambda F}{\zeta}-\frac{\zeta}{2 \lambda^{2}}
\end{gathered}
$$

Substituting Eq. (55) into Eq. (56) to delete $C_{1}$ gives

$$
\zeta=\left[\left(\sin ^{-2} \theta_{n}+1\right)^{1 / 2}-\sin ^{-1} \theta_{n}\right]\left(2 F \lambda^{3}\right)^{1 / 2}
$$

The boundary condition at $y=1$ then becomes

$$
\begin{gathered}
\text { (a) } \frac{F^{2}}{\bar{C}_{1}} \cot ^{2} \theta_{m}=n_{0} \\
\text { (b) } \frac{\left(2 \bar{C}_{1}\right)^{3 / 2}}{F^{2}}=(1+\nu) \frac{\cos ^{3} \theta_{m}}{\sin ^{2} \theta_{m}}+\frac{\bar{C}_{1} n_{0}(1-\nu)}{F^{2}} \cos \theta_{m}
\end{gathered}
$$

Substituting Eq. (55) into Eqs. (58) and (59) gives

$$
\text { (a) } \frac{\cot ^{2} \theta_{m}}{\cot ^{2} \theta_{n}}=\frac{n_{0}}{\lambda F}
$$




$$
\text { (b) }\left(\frac{8}{F \lambda^{3}}\right)^{1 / 2} \cot ^{3} \theta_{n}=(1+\nu) \frac{\cos ^{3} \theta_{m}}{\sin ^{2} \theta_{m}}+\frac{n_{0}(1-\nu)}{\lambda F} \cos \theta_{m} \cot ^{2} \theta_{n}
$$

Substituting Eqs. (55)-(57) into Eq. (54) gives

$$
\begin{gathered}
\left(\sin ^{-2} \theta_{n}+1\right)^{1 / 2}-\sin ^{-1} \theta_{n}+\frac{\sin ^{3} \theta_{n}}{2 \cos ^{3} \theta_{n}}\left[\frac{\cos \theta_{m}}{\sin ^{2} \theta_{m}}-\ln \left(\cot \frac{\theta_{m}}{2}\right)\right. \\
\left.-\frac{\cos \theta_{n}}{\sin ^{2} \theta_{n}}+\ln \left(\cot \frac{\theta_{n}}{2}\right)\right]=\left(2 F \lambda^{3}\right)^{-1 / 2}
\end{gathered}
$$

Substituting Eq. (51) into Eq. (24) and making use of Eq. (52) give

$$
\frac{w_{0}}{h}=\left(2 \bar{C}_{1}\right)^{-1 / 2} F\left[\ln \left|\frac{1}{\sin \theta_{n}}-\frac{\cos \theta_{n}}{\sin \theta_{n}}\right|-\ln \left|\frac{1}{\sin \theta_{m}}-\frac{\cos \theta_{m}}{\sin \theta_{m}}\right|\right]
$$

By solving Eqs. (60)-(62), $\theta_{n}$ and $\theta_{m}$ can be obtained. It can then be used to determine $w_{0}$ and $d$ by making use of Eqs. (63) and (47).

Based on Eq. (51), we can obtain

$$
\begin{gathered}
\frac{8 a^{2} N_{r}}{E h^{3}}=\frac{F^{2}}{\bar{C}_{1}} \frac{\cot ^{2} \theta}{y} \\
\frac{8 a^{2} N_{\theta}}{E h^{3}}=\frac{F^{2}}{\bar{C}_{1}}\left[\frac{\left.\left(2 \bar{C}_{1}\right)\right)^{3 / 2}}{F^{2}} \frac{1}{\cos \theta}-\frac{\cos ^{2} \theta}{y \sin ^{2} \theta}\right]
\end{gathered}
$$

To ensure that $N_{\theta} \geq 0$ in $\zeta<y<1$, we substitute Eq. (53) into Eq. (65) and obtain the following relationship:

$$
-\zeta \leq C_{2} \leq \zeta\left[\frac{2}{\cos ^{2} \theta_{n}}-2 \ln \left(\cot \frac{\theta_{n}}{2}\right) \frac{\sin ^{2} \theta_{n}}{\cos ^{3} \theta_{n}}-1\right]
$$

- Branch 3: $C_{1}=0$

Integrating both sides of Eq. (33) gives

$$
S=\left(\frac{9}{2} F^{2}\right)^{1 / 3}\left(y+C_{2}\right)^{2 / 3}
$$

where $C_{2}$ is an integration constant. Substituting Eq. (67) into Eq. (24) gives

$$
\frac{w_{0}}{h}=\left(\frac{3}{4} F^{2}\right)^{1 / 3}\left[\left(1+C_{2}\right)^{1 / 3}-\left(\zeta+C_{2}\right)^{1 / 3}\right]
$$


Eqs. (31) and (32) can then be transformed into the following equations:

$$
\begin{aligned}
\zeta & =(\sqrt{2}-1)\left(2 F \lambda^{3}\right)^{1 / 2} \\
C_{2} & =\left(\frac{4}{3}-\sqrt{2}\right)\left(2 F \lambda^{3}\right)^{1 / 2}
\end{aligned}
$$

The boundary condition at $y=1$ becomes:

$$
\begin{gathered}
\text { (a) } \xi=\left(\frac{3}{4}\right)^{2 / 3}\left(1+C_{2}\right)^{2 / 3} \\
\text { (b) } \xi=\left(\frac{3}{4}\right)^{2 / 3}\left(1+C_{2}\right)^{-1 / 3}\left[\frac{3 \nu-1}{3(\nu-1)}+\frac{\nu+1}{\nu-1} C_{2}\right]
\end{gathered}
$$

Based on Eq. (67) we can obtain

$$
\begin{gathered}
\frac{4 a^{2} N_{r}}{E h^{3}}=\left(\frac{3}{4} F\right)^{2 / 3} \frac{\left(y+C_{2}\right)^{2 / 3}}{y} \\
\frac{4 a^{2} N_{\theta}}{E h^{3}}=\left(\frac{3}{4} F\right)^{2 / 3}\left(y+C_{2}\right)^{-1 / 3}\left(\frac{1}{3}-\frac{C_{2}}{y}\right)
\end{gathered}
$$

To ensure that $N_{\theta} \geq 0$ in $\zeta<y<1$, we have $-\zeta \leq C_{2} \leq \zeta / 3$.

\section{Summary of the Exact Solution}

The complete solution can be summarized as shown in Table 2. For a circular membrane with boundary condition $(a)$, the spherical indentation problem has the following solution from Branch $C_{1}>0$ :

$$
\left\{\begin{array}{l}
\frac{\sin \theta_{m}}{\sin \theta_{n}}=\left(2 F \lambda^{3}\right)^{-1 / 2}\left(\lambda \sqrt{2 n_{0}}\right) \\
\left(\cos ^{2} \theta_{n}+1\right)^{1 / 2}-\cos \theta_{n}+\frac{2 \theta_{m}-\sin 2 \theta_{m}-2 \theta_{n}+\sin 2 \theta_{n}}{4 \sin ^{3} \theta_{n}}=\left(2 F \lambda^{3}\right)^{-1 / 2}
\end{array}\right.
$$

when $0<\xi^{3 / 2}<\xi^{*}$ with $\xi^{*}=3 / 4+(1-3 \sqrt{2} / 4)\left(2 F \lambda^{3}\right)^{1 / 2}$; and it has the following solution from Branch $C_{1}<0$ :

$$
\left\{\begin{array}{l}
\frac{\cot \theta_{m}}{\cot \theta_{n}}=\left(2 F \lambda^{3}\right)^{-1 / 2}\left(\lambda \sqrt{2 n_{0}}\right) \\
\left(\sin ^{-2} \theta_{n}+1\right)^{1 / 2}-\sin ^{-1} \theta_{n}+\frac{\sin ^{3} \theta_{n}}{2 \cos ^{3} \theta_{n}}\left[\frac{\cos \theta_{m}}{\sin ^{2} \theta_{m}}-\ln \left(\cot \frac{\theta_{m}}{2}\right)\right. \\
\left.-\frac{\cos \theta_{n}}{\sin ^{2} \theta_{n}}+\ln \left(\cot \frac{\theta_{n}}{2}\right)\right]=\left(2 F \lambda^{3}\right)^{-1 / 2}
\end{array}\right.
$$


when $\xi^{3 / 2}>\xi^{*}$.

For the problem with boundary condition (b), it has the following solution from Branch $C_{1}>0:$ :

$$
\left\{\begin{array}{l}
4\left(2 F \lambda^{3}\right)^{-1 / 2} \sin ^{3} \theta_{n}=(1+\nu) \frac{\sin ^{3} \theta_{m}}{\cos \theta_{m}}+\left[\left(2 F \lambda^{3}\right)^{-1 / 2}\left(\lambda \sqrt{2 n_{0}}\right)\right]^{2}(1-\nu) \frac{\sin ^{2} \theta_{n} \sin \theta_{m}}{\cos \theta_{m}} \\
\left(\cos ^{2} \theta_{n}+1\right)^{1 / 2}-\cos \theta_{n}+\frac{2 \theta_{m}-\sin 2 \theta_{m}-2 \theta_{n}+\sin 2 \theta_{n}}{4 \sin ^{3} \theta_{n}}=\left(2 F \lambda^{3}\right)^{-1 / 2}
\end{array}\right.
$$

when $0<\nu<1-\nu^{*}$ with $\nu^{*}=\left[2+(8-6 \sqrt{2})\left(2 F \lambda^{3}\right)^{1 / 2}\right] /\{3+(4-$ $\left.3 \sqrt{2})\left(2 F \lambda^{3}\right)^{1 / 2}-3 \xi(4 / 3)^{2 / 3}\left[1+(4 / 3-\sqrt{2})\left(2 F \lambda^{3}\right)^{1 / 2}\right]^{1 / 3}\right\}$; and it has the following solution from Branch $C_{1}<0$ :

$$
\left\{\begin{array}{l}
4\left(2 F \lambda^{3}\right)^{1 / 2} \cot ^{3} \theta_{n}=(1+\nu) \frac{\cos ^{3} \theta_{m}}{\sin ^{2} \theta_{m}}+\left[\left(2 F \lambda^{3}\right)^{-1 / 2}\left(\lambda \sqrt{2 n_{0}}\right)\right]^{2}(1-\nu) \cos \theta_{m} \cot ^{2} \theta_{n} \\
\left(\sin ^{-2} \theta_{n}+1\right)^{1 / 2}-\sin ^{-1} \theta_{n}+\frac{\sin ^{3} \theta_{n}}{2 \cos ^{3} \theta_{n}}\left[\frac{\cos \theta_{m}}{\sin ^{2} \theta_{m}}-\ln \left(\cot \frac{\theta_{m}}{2}\right)\right. \\
\left.-\frac{\cos \theta_{n}}{\sin ^{2} \theta_{n}}+\ln \left(\cot \frac{\theta_{n}}{2}\right)\right]=\left(2 F \lambda^{3}\right)^{-1 / 2}
\end{array}\right.
$$

when $1-\nu^{*}<\nu<0.5$.

In Branch $C_{1}>0$, by solving Eq. (75) or Eq. (77), $\theta_{n}$ and $\theta_{m}$ can be obtained. It can then be used to determine $\zeta, \epsilon_{m}$ and $d$, where

$$
\begin{gathered}
\zeta\left(2 F \lambda^{3}\right)^{-1 / 2}=\left(\cos ^{2} \theta_{n}+1\right)^{1 / 2}-\cos \theta_{n} \\
\frac{2 \lambda d}{h}\left(2 F \lambda^{3}\right)^{-1 / 2}=\frac{\theta_{m}-\theta_{n}}{\sin \theta_{n}}+\left(\cos ^{2} \theta_{n}+1\right)^{1 / 2}-\cos \theta_{n} \\
\epsilon_{m}=\frac{1-\nu}{16}\left(\frac{a}{R}\right)^{2}\left(\frac{2 F \lambda^{3}}{\zeta}+\zeta\right)
\end{gathered}
$$

In Branch $C_{1}<0$, by solving Eq. (76) or Eq. (78), we get $\theta_{n}$ and $\theta_{m}$. The values of $\zeta$ and $d$ can be obtained from

$$
\begin{aligned}
& \zeta\left(2 F \lambda^{3}\right)^{-1 / 2}=\left(\sin ^{-2} \theta_{n}+1\right)^{1 / 2}-\sin ^{-1} \theta_{n} \\
& \frac{2 \lambda d}{h}\left(2 F \lambda^{3}\right)^{-1 / 2}=\frac{1}{\cot \theta_{n}}\left[\ln \left|\frac{1}{\sin \theta_{n}}-\frac{\cos \theta_{n}}{\sin \theta_{n}}\right|-\ln \left|\frac{1}{\sin \theta_{m}}-\frac{\cos \theta_{m}}{\sin \theta_{m}}\right|\right] \\
& +\left(\sin ^{-2} \theta_{n}+1\right)^{1 / 2}-\sin ^{-1} \theta_{n}
\end{aligned}
$$


The maximum indentation strain $\epsilon_{m}$ can then be obtained by substituting Eq. (82) into Eq. (81).

In Branch $C_{1}=0$, explicit analytical solutions are directly obtained as follows:

$$
\begin{gathered}
\zeta\left(2 F \lambda^{3}\right)^{-1 / 2}=\sqrt{2}-1 \\
\frac{2 \lambda d}{h}\left(2 F \lambda^{3}\right)^{-1 / 2}=\left[3\left(2 F \lambda^{3}\right)^{-1 / 2}+4-3 \sqrt{2}\right]^{1 / 3}+\sqrt{2}-2 \\
\epsilon_{m}=\frac{1-\nu}{4 \sqrt{2}}\left(\frac{a}{R}\right)^{2}\left(2 F \lambda^{3}\right)^{1 / 2}
\end{gathered}
$$

which are valid when $\xi^{3 / 2}=\xi^{*}$ for the problems with boundary condition $(a)$, and $\nu=1-\nu^{*}$ for the problems with boundary condition $(b)$.

When the indenter radius is much smaller than that of the circular membrane, i.e., $R / a \rightarrow 0$, the applied force can be assumed as a point load. In this special case, our exact solution converges to the Schwerin-type pointload solution (Schwerin, 1929; Komaragiri et al., 2005; Jin and Wang, 2008). The complete point-load solution can be obtained by making $C_{2}=0$ in every branch (Jin and Wang, 2008), and the result is summarized in Table 3. For a circular membrane with boundary condition $(a)$, it has the following solution from Branch $C_{1}>0$ :

$$
\left\{\begin{array}{l}
\left(\frac{d}{h}\right)^{3}=\frac{\theta_{m}^{3}}{2 \theta_{m}-\sin 2 \theta_{m}} F \\
\xi^{3 / 2}=\frac{\sin ^{3} \theta_{m}}{2 \theta_{m}-\sin 2 \theta_{m}}
\end{array}\right.
$$

when $0<\xi^{3 / 2}<3 / 4$; and it has the following solution from Branch $C_{1}<0$ :

$$
\left\{\begin{array}{l}
\left(\frac{d}{h}\right)^{3}=-\frac{1}{2}\left[\frac{\cos \theta_{m}}{\sin ^{2} \theta_{m}}-\ln \left(\cot \frac{\theta_{m}}{2}\right)\right]^{-1} \ln ^{3}\left|\frac{1}{\sin \theta_{m}}-\frac{\cos \theta_{m}}{\sin \theta_{m}}\right| F \\
\xi^{3 / 2}=\frac{\cot ^{3} \theta_{m}}{2\left[\frac{\cos \theta_{m}}{\sin ^{2} \theta_{m}}-\ln \left(\cot \frac{\theta_{m}}{2}\right)\right]}
\end{array}\right.
$$

when $\xi^{3 / 2}>3 / 4$. The curves of central deflection versus load can be obtained by generating a parametric plot by varying the parameter $\theta_{m}$. 
For the problem with boundary condition $(b)$, it has the following solution from Branch $C_{1}>0$ :

$$
\left\{\begin{array}{l}
\left(\frac{d}{h}\right)^{3}=\frac{\theta_{m}^{3}}{2 \theta_{m}-\sin 2 \theta_{m}} F \\
\nu=1+\frac{2 \theta_{m} \cos \theta_{m}-\sin 2 \theta_{m} \cos \theta_{m}-2 \sin ^{3} \theta_{m}}{\sin ^{3} \theta_{m}-\xi \sin \theta_{m}\left(2 \theta_{m}-\sin 2 \theta_{m}\right)^{2 / 3}}
\end{array}\right.
$$

when $0<\nu<1-\nu^{* *}$ with $\nu^{* *}=2 /\left[3-3(4 / 3)^{2 / 3} \xi\right]$; and it has the following solution from Branch $C_{1}<0$ :

$$
\left\{\begin{array}{l}
\left(\frac{d}{h}\right)^{3}=-\frac{1}{2}\left[\frac{\cos \theta_{m}}{\sin ^{2} \theta_{m}}-\ln \left(\cot \frac{\theta_{m}}{2}\right)\right]^{-1} \ln ^{3}\left|\frac{1}{\sin \theta_{m}}-\frac{\cos \theta_{m}}{\sin \theta_{m}}\right| F \\
\nu=1+\frac{2\left[\cos \theta_{m}-\sin ^{2} \theta_{m} \ln \left(\cot \frac{\theta_{m}}{2}\right)-\cos ^{3} \theta_{m}\right]}{\cos ^{3} \theta_{m}-2^{\frac{2}{3}} \xi \cos \theta_{m}\left[\cos \theta_{m} \sin \theta_{m}-\sin ^{3} \theta_{m} \ln \left(\cot \frac{\theta_{m}}{2}\right)\right]^{\frac{2}{3}}}
\end{array}\right.
$$

when $1-\nu^{* *}<\nu<0.5$.

In Branch $C_{1}=0$, explicit analytical solutions are directly obtained as follows:

$$
\left(\frac{d}{h}\right)^{3}=\frac{3}{4} F=\frac{3 a^{2} P_{0}}{\pi E h^{4}}
$$

which is the well-known Schwerin solution (Schwerin, 1929). It is valid only when $\xi^{3 / 2}=3 / 4$ for the problems with boundary condition $(a)$, or $\nu=1-\nu^{* *}$ for the problems with boundary condition $(b)$. This is consistent with Jin and Wang (2008)'s conclusion that Schwerin's one-term particular solution is strictly valid only at zero prestretch for $\nu=1 / 3$, or a certain value of prestretch for a given Poisson's ratio.

\section{Results and Discussion}

It can be seen that although our solutions are exact, their expressions are very complicated and cast in a parametric form. This is an important limitation of the current work. For ease of application, we here developed an analytical solution in explicit form for the special point-load case. For the general case, the MATLAB source codes used to generate the exact solution have been provided in Supplementary Information ${ }^{1}$. With the provided

\footnotetext{
${ }^{1}$ The MATLAB source codes (with detailed explanatory comments) used to plot Fig. 2, Fig. 3, Fig. 5, Fig. 6, and Fig. 7 have been provided in Supplementary Information.
} 
MATLAB codes, the users just need to specify the Young's modulus and the Poisson's ratio of the membrane, the radius and the thickness of the membrane, the radius of the spherical indenter, and the prescribed radial stress/displacement at the boundary, and then the codes will generate the load-deflection curves.

For the spherical indentation problem with boundary condition (a), the solution from Branch $C_{1}>0$ and Branch $C_{1}<0$ can be written in the following form, respectively:

$$
\begin{aligned}
& \left\{\begin{array}{l}
\frac{\sin \theta_{m}}{\sin \theta_{n}}-\left(\lambda \sqrt{2 n_{0}}\right)\left[\left(\cos ^{2} \theta_{n}+1\right)^{1 / 2}-\cos \theta_{n}+\frac{2 \theta_{m}-\sin 2 \theta_{m}-2 \theta_{n}+\sin 2 \theta_{n}}{4 \sin ^{3} \theta_{n}}\right]=0 \\
\left(2 F \lambda^{3}\right)^{-1 / 2}=\left(\cos ^{2} \theta_{n}+1\right)^{1 / 2}-\cos \theta_{n}+\frac{2 \theta_{m}-\sin 2 \theta_{m}-2 \theta_{n}+\sin 2 \theta_{n}}{4 \sin ^{3} \theta_{n}}
\end{array}\right. \\
& \left\{\begin{array}{l}
\frac{\cot \theta_{m}}{\cot \theta_{n}}-\left(\lambda \sqrt{2 n_{0}}\right)\left\{\left(\sin ^{-2} \theta_{n}+1\right)^{1 / 2}-\sin ^{-1} \theta_{n}+\frac{\sin ^{3} \theta_{n}}{2 \cos ^{3} \theta_{n}}\left[\frac{\cos \theta_{m}}{\sin ^{2} \theta_{m}}-\ln \left(\cot \frac{\theta_{m}}{2}\right)\right.\right. \\
\left.\left.-\frac{\cos \theta_{n}}{\sin ^{2} \theta_{n}}+\ln \left(\cot \frac{\theta_{n}}{2}\right)\right]\right\}=0 \\
\left(2 F \lambda^{3}\right)^{-1 / 2}=\left(\sin ^{-2} \theta_{n}+1\right)^{1 / 2}-\sin ^{-1} \theta_{n}+\frac{\sin ^{3} \theta_{n}}{2 \cos ^{3} \theta_{n}}\left[\frac{\cos \theta_{m}}{\sin ^{2} \theta_{m}}-\ln \left(\cot \frac{\theta_{m}}{2}\right)\right. \\
\left.-\frac{\cos \theta_{n}}{\sin ^{2} \theta_{n}}+\ln \left(\cot \frac{\theta_{n}}{2}\right)\right]
\end{array}\right.
\end{aligned}
$$

For every $\theta_{n} \quad\left(0<\theta_{n}<\pi / 2\right), \theta_{m}$ can be obtained from the first equation in Eq. (92) and Eq. (93). We can then substitute $\theta_{m}$ and $\theta_{n}$ into the second equation in Eq. (92) and Eq. (93) to obtain $\left(2 F \lambda^{3}\right)^{-1 / 2}$. The central deflection can then be obtained by substituting $\theta_{m}$ and $\theta_{n}$ into Eq. (80) and Eq. (83). Similarly, the contact radius can be obtained by substituting $\theta_{m}$ and $\theta_{n}$ into Eq. (79) and Eq. (82). The relationship among load, central deflection, and contact radius has been plotted in Fig. 2(a). It can be seen that for this case, the Poisson's ratio of the membrane does not influence the relationship among load, central deflection, and contact radius. The normalized load $\left(2 F \lambda^{3}\right)^{-1 / 2}$ depends on both the normalized central deflection $\lambda d / h$ and the normalized pre-tension $\lambda\left(2 n_{0}\right)^{1 / 2}$, where $\left(2 F \lambda^{3}\right)^{-1 / 2}=\left[8 P_{0} R^{3} /\left(\pi E h a^{4}\right)\right]^{-1 / 2}$, $\lambda d / h=R d / a^{2}$, and $\lambda\left(2 n_{0}\right)^{1 / 2}=4 R\left(N_{0} / E h\right)^{1 / 2} / a$. Pre-tension tends to stiffen the membrane and leads to a reduction in the deflection, i.e., as the pre-tension increases, the deflection becomes smaller for a certain amount of load. The contact radius increases with increasing central deflection, and as the pre-tension increases, the contact radius becomes larger for a certain 
amount of central deflection.

For the problem with boundary condition (b), in the limit of zero prestrain, the solution from Branch $C_{1}>0$ and Branch $C_{1}<0$ can be written in the following form, respectively:

$$
\begin{aligned}
& \left\{\begin{array}{l}
(1+\nu) \frac{\sin ^{3} \theta_{m}}{\cos \theta_{m}}-2 \theta_{m}+\sin 2 \theta_{m}=2 \sin ^{3} \theta_{n}\left[\left(\cos ^{2} \theta_{n}+1\right)^{1 / 2}-\cos \theta_{n}\right]-2 \theta_{n}+\sin 2 \theta_{n} \\
\left(2 F \lambda^{3}\right)^{-1 / 2}=\left(\cos ^{2} \theta_{n}+1\right)^{1 / 2}-\cos \theta_{n}+\frac{2 \theta_{m}-\sin 2 \theta_{m}-2 \theta_{n}+\sin 2 \theta_{n}}{4 \sin ^{3} \theta_{n}}
\end{array}\right. \\
& \left\{\begin{array}{l}
(1+\nu) \frac{\cos ^{3} \theta_{m}}{\sin ^{2} \theta_{m}}-2 \frac{\cos \theta_{m}}{\sin ^{2} \theta_{m}}+2 \ln \left(\cot \frac{\theta_{m}}{2}\right)=4 \cot ^{3} \theta_{n}\left[\left(\sin ^{-2} \theta_{n}+1\right)^{1 / 2}-\sin ^{-1} \theta_{n}\right] \\
-2 \frac{\cos \theta_{n}}{\sin ^{2} \theta_{n}}+2 \ln \left(\cot \frac{\theta_{n}}{2}\right) \\
\left(2 F \lambda^{3}\right)^{-1 / 2}=\left(\sin ^{-2} \theta_{n}+1\right)^{1 / 2}-\sin ^{-1} \theta_{n}+\frac{\sin ^{3} \theta_{n}}{2 \cos ^{3} \theta_{n}}\left[\frac{\cos \theta_{m}}{\sin ^{2} \theta_{m}}-\ln \left(\cot \frac{\theta_{m}}{2}\right)\right. \\
\left.-\frac{\cos \theta_{n}}{\sin ^{2} \theta_{n}}+\ln \left(\cot \frac{\theta_{n}}{2}\right)\right]
\end{array}\right.
\end{aligned}
$$

For every $\theta_{n} \quad\left(0<\theta_{n}<\pi / 2\right), \theta_{m}$ can be obtained from the first equation in Eq. (94) and Eq. (95). We can then substitute $\theta_{m}$ and $\theta_{n}$ into the second equation in Eq. (94) and Eq. (95) to obtain $\left(2 F \lambda^{3}\right)^{-1 / 2}$. The central deflection can then be obtained by substituting $\theta_{m}$ and $\theta_{n}$ into Eq. (80) and Eq. (83). Similarly, the contact radius can be obtained by substituting $\theta_{m}$ and $\theta_{n}$ into Eq. (79) and Eq. (82). The relationship among load, central deflection, and contact radius has been plotted in Fig. 2(b). It can be seen that for this case, the normalized load $\left(2 F \lambda^{3}\right)^{-1 / 2}$ depends on both the normalized central deflection $\lambda d / h$ and the Poisson's ratio of the membrane. A larger value of Poisson's ratio gives a smaller deflection and a smaller contact radius for a certain amount of load.

For the problem with boundary condition (b), in the limit of zero prestrain, the approximate solution provided by Begley and Mackin (2004) is given by Eq. (4), which can be written in the following form:

$$
\left(\frac{R d}{a^{2}}\right)^{3}=\frac{16}{9 \pi}\left(\frac{a}{R}\right)^{1 / 4}\left(\frac{P_{0} R^{3}}{E h a^{4}}\right)
$$

According to the approximate solution, the normalized load $P_{0} R^{3} /\left(E h a^{4}\right)$ does not depend on the Poisson's ratio of the membrane, but depends on the normalized central deflection $R d / a^{2}$ and the ratio of the two radii $R / a$. 
Fig. 3(a) compares the load-deflection curves based on the approximate solution and the exact solution. It can be seen that there is a significant difference between the two solutions. The approximate solution predicts a much larger central deflection than the exact solution for a given amount of load.

The problem with boundary condition (b) with nonzero prestrain can be solved in a similar manner. The normalized load $\left(2 F \lambda^{3}\right)^{-1 / 2}$ depends on the normalized central deflection $\lambda d / h$, the Poisson's ratio $\nu$, and the normalized pre-tension $\lambda\left(2 n_{0}\right)^{1 / 2}$. For the case when $\lambda\left(2 n_{0}\right)^{1 / 2}=0.9$, the relationship among load, central deflection, and contact radius has been plotted in Fig. 2(c). It can be seen that a larger value of Poisson's ratio or pre-tension gives a smaller deflection and a smaller contact radius for a certain amount of load.

When the prestrain is not zero, the approximate solution provided by Begley and Mackin (2004) is given by Eq. (3). Fig. 3(b) compares the loaddeflection curves for the case when $\lambda\left(2 n_{0}\right)^{1 / 2}=0.9$ and $R / a=0.3$ based on the approximate solution and the exact solution. Fig. 3(c) plots the case when $\lambda\left(2 n_{0}\right)^{1 / 2}=0.9$ and $R / a=1.0$. Again, there is a significant difference between the two solutions even when a larger indenter is used. According to the approximate solution, a larger value of Poisson's ratio gives a larger central deflection for a certain amount of load, which is the opposite trend of that predicted by the exact solution. Moreover, the approximate solution predicts a nonzero value of indenter load when the central deflection is zero, which is not realistic.

For the point-load problem with boundary condition (a), the solution can be written in the following form:

$$
\left(\frac{d}{h}\right)^{3}=g_{1}(\xi) F
$$

where $g_{1}(\xi) \approx 0.1782 \xi^{4}-1.191 \xi^{3}+3.106 \xi^{2}-4.018 \xi+2.537$, as plotted in Fig. 4(a). Again, for this case, the Poisson's ratio of the membrane does not influence the load-deflection relationship. Pre-tension tends to stiffen the membrane and leads to a reduction in the deflection.

For the point-load problem with boundary condition (b), the solution can be written in the following form:

$$
\left(\frac{d}{h}\right)^{3}=g_{2}(\xi, \nu) F
$$

Fig. 4(b) shows the plot of $g_{2}(\xi, \nu)$ as a function of $\nu$ when $\xi=0.0,0.2$, 0.4 , and 0.6. It shows that a larger value of Poisson's ratio gives a smaller 
deflection for a certain amount of load. Again, pre-tension tends to stiffen the membrane, i.e., as the pre-tension increases, the deflection becomes smaller and more indifferent to the change of Poisson's ratio.

Note that the exact analytical solution presented in this study converges to the point-load solution as $R / a \rightarrow 0$, but the approximate solution provided by Begley and Mackin (2004) does not. For example, for the point-load problem with boundary condition (b), in the limit of zero prestrain, the exact solution can be written as:

$$
\left(\frac{d}{h}\right)^{3}=\frac{4}{\pi} g_{2}(\nu)\left(\frac{P_{0} a^{2}}{E h^{4}}\right)
$$

where $g_{2}(\nu) \approx-0.2499 \nu^{2}-0.3999 \nu+0.9109$. The approximate solution, i.e. Eq. (4), can be written in the following form:

$$
\left(\frac{d}{h}\right)^{3}=\frac{16}{9 \pi}\left(\frac{a}{R}\right)^{1 / 4}\left(\frac{P_{0} a^{2}}{E h^{4}}\right)
$$

It can be seen that as $R / a \rightarrow 0$, the exact solution predicts a finite deflection, whereas the approximate solution predicts an infinite deflection. The result of infinite deflection under point load is true for neo-Hookean membrane (Yang and Feng, 1970; Yang and Hsu, 1971; Fulton and Simmonds, 1986; Nadler and Tang, 2008), but not realistic for Föppl-Hecky membrane. However, the approximate solution is derived based on Föppl-Hecky theory (Begley and Mackin, 2004), so this result is not self-consistent.

\section{Comparison with Experimental Results}

In this section, experiments of spherical indentation has been performed, and the exact analytical solution presented in this study is compared with experimental results from existing literature as well as our own experimental results.

Experimental data have been provided by Bhatia and Nachbar (1968) for the load-deflection characteristics of a Mylar sheet stretched in its plane by deadweight loading, which corresponds to the problem with boundary condition (a). The Mylar sheet has the following properties and dimensions: $E=4.62 \mathrm{GPa}, \nu=0.3, h=15.24 \mu \mathrm{m}$, and $a=127 \mathrm{~mm}$. The transverse load $P_{0}$ was applied at the center of the sheet by an indenter having a hemispherical tip of radius $R=0.0125 a$. Experimental results are presented for three different values of prescribed radial stress, $N_{0}$, at the boundary: 89.3 
$\mathrm{N} / \mathrm{m}, 137.6 \mathrm{~N} / \mathrm{m}$, and $263.7 \mathrm{~N} / \mathrm{m}$. Fig. 5 plots the load-deflection curves and provides a comparison of the analytical solutions with the experimental data. It can be seen that our exact solution based on Eq. (80) and Eq. (83) matches the experimental data very well, however, the approximate solution provided by Begley and Mackin (2004) based on Eq. (3) does not match the experimental data. This is because during the derivation of the approximate solution (Begley and Mackin, 2004), boundary condition (b) is always assumed and boundary condition (a) is never considered. We can also see that the approximate solution predicts a nonzero value of indenter load when the central deflection is zero, which is never observed in the experiments.

Experimental data have been provided by Scott et al. (2004) for the loaddeflection characteristics of an elastomer film with fixed radial displacement at the boundary, which corresponds to the problem with boundary condition (b). The prestress is negligibly small, far below what can be reliably measured with nanometer displacement and nano-Newton load resolution. The elastomer film has the following properties and dimensions: $E=1.15$ MPa, $\nu=0.5, h=127 \mu \mathrm{m}$, and $a=25.4 \mathrm{~mm}$. The Young's modulus is measured from a conventional uni-axial test. The transverse load $P_{0}$ was applied at the center of the film by indenters having a hemispherical tip with different $R / a$ ratios: $R / a=0.03, R / a=0.14, R / a=0.50$, and $R / a=1.00$. Fig. 6 plots the load-deflection curves and provides a comparison of the analytical solutions with the experimental data. It can be seen that increasing $R / a$ decreases the central deflection for a given amount of load. A quantitative comparison among the experimental data, the approximate solution, and the exact solution has been provided in Table 4 . It can be seen that when the indentation depth is low, such as when $d=2.00 \mathrm{~mm}$, neither the approximate solution nor the exact solution shows a good match with the experimental data, but the agreement between our exact solution based on Eq. (80) and Eq. (83) and the experimental data is better than that between the approximate solution provided by Begley and Mackin (2004) and the experimental data. As the indentation depth increases, such as when $d=4.00$ $\mathrm{mm}$, the exact solution shows a fairly good agreement with the experimental data, whereas the approximate solution still significantly deviates from the experimental data. ${ }^{2}$

\footnotetext{
${ }^{2}$ We here have to point out that the plots in Fig. 7 provided by Scott et al. (2004), showing that the approximate solution matches the experimental data, are not correct.
} 
We also performed experiments of spherical indentation on freestanding circular sheets made of polyethylene terephthalate (PET) and compared the existing solutions with the experimental results. While complete details of the experimental set-up are provided by Jin et al. (2016), we here provide a brief overview. ${ }^{3}$ The test arrangement is a circular freestanding PET sheet with fixed displacement at the boundary that is indented by finitesized spheres. The prestress is negligibly small. A brass spherical indenter is pressed into the sheet using a positioning stage that provides an independent displacement measurement. Loads are measured using a conventional load cell. Load-deflection curves are presented for a single thickness, $h=12 \mu \mathrm{m}$, and span, $a=37.45 \mathrm{~mm}$, and four different indenter sizes with $R / a=0.17$, $R / a=0.25, R / a=0.34$, and $R / a=0.46$, respectively, as shown in Fig. 7 . According to the manufacturer, the Young's modulus and Poisson's ratio of the PET sheet are $E=3.0 \pm 0.2 \mathrm{GPa}$ and $\nu=0.4 \pm 0.03$, respectively. The exact solution and the approximate solution are both plotted based on $E=3.2 \mathrm{GPa}$ and $\nu=0.4$. It can be seen that a good agreement can be obtained between the exact solution and the experimental data. Fig. 6 and Fig. 7 also show that even when larger indenters are used, the approximate solution still significantly deviates from the experimental data.

\section{Concluding Remarks}

To provide a more accurate solution to predict the relationship among contact radius, load and deflection, the problem of spherical indentation of a freestanding circular membrane has been revisited. This work provided an exact analytical solution to the governing equations of Föppl-Hecky membrane indented by a frictionless spherical indenter. It has been found that due to their approximate nature, the solutions from existing literature are not accurate enough under a wide range of conditions, and using the approximate solutions to extract mechanical properties from indentation testing of freestanding films may result in significant errors. Due to the development of novel micro-fabrication techniques to produce ultra-thin materials, such as carbon nanotubes and graphene (Gomez-Navarro et al., 2008; Lee et al.,

\footnotetext{
${ }^{3}$ This experimental set-up was designed for the purpose of spherical indentation testing of separator membranes of lithium-ion batteries. We also used it to test PET sheets. The experimental data on PET sheets are published here, whereas the experimental data on separator membranes will be published by Jin et al. (2016).
} 
2008; Poot and van der Zant, 2008; Duan and Wang, 2009; Chen et al., 2015; Cao et al., 2016), and increasing interest in thin biological membranes, such as cornea (Ahearne et al., 2007) and lung surfactant (Alonso et al., 2004), in recent years, the mechanical characterization of thin films has received a significant amount of attention. The exact solution provided by the current study is bound to have important applications.

\section{Acknowledgment}

C. Jin thanks the start-up funds provided by the Department of Mechanical Engineering at the State University of New York at Binghamton. J. Li thanks the funding support from the US-China CERC-CVC under the Award Number DE-PI0000012 and the experiment was conducted at Oak Ridge National Laboratory, managed by UT Battelle, LLC, for the U. S. Department of Energy under contract DE-AC05-00OR22725.

\section{References}

Ahearne, M., Yang, Y., Then, K.Y., Liu, K.K., 2007. An indentation technique to characterize the mechanical and viscoelastic properties of human and porcine corneas. Ann. Biomed. Eng. 35, 1608-1616.

Alonso, C., Alig, T., Yoon, J., Bringezu, F., Warriner, H., Zasadzinski, J.A., 2004. More than a monolayer: relating lung surfactant structure and mechanics to composition. Biophys. J. 87, 4188-4202.

Begley, M.R., Mackin, T.J., 2004. Spherical indentation of freestanding circular films in the membrane regime. J. Mech. Phys. Solids 52, 2005-2023.

Ben-David, E., Tepper-Faran, T., Rittel, D., Shilo, D., 2014. A new methodology for uniaxial tensile testing of free-standing thin films at high strainrates. Exp. Mech. 54, 1687-1696.

Bhatia, N.M., Nachbar, W., 1968. Finite indentation of an elastic membrane by a spherical indenter. Int. J. Non-Linear Mech. 3, 307-324.

Cao, Z., Tao, L., Akinwande,D., Huang, R., Liechti, K.M., 2016. Mixed-mode traction-separation relations between graphene and copper by blister tests. International Journal of Solids and Structures 84, 147-159. 
Chen, X., Yi, C., Ke, C., 2015. Bending stiffness and interlayer shear modulus of few-layer graphene. Appl. Phys. Lett. 106, 101907.

Chua, W.K., Oyen, M.L., 2009. Do we know the strength of the chorioamnion? A critical review and analysis. European Journal of Obstetrics \& Gynecology and Reproductive Biology 144, S128-S133.

Duan, W.H., Wang, C.M., 2009. Nonlinear bending and stretching of a circular graphene sheet under a central point load. Nanotechnology 20, 075702.

Espinosa, H.D., Prorok, B.C., 2001. Effects of film thickness on the yielding behavior of polycrystalline gold films. Mater. Res. Soc. Symp. Proc. 695, 349-354.

Espinosa, H.D., Prorok, B.C., Fischer, M., 2003. A methodology for determining mechanical properties of freestanding thin films and MEMS materials. J. Mech. Phys. Solids 51, 47-67.

Föppl, A., 1907. Vorlesungen über technische Mechanik, Bd. 3. B.G. Teubner, Leipzig.

Fulton, J.P., Simmonds, J.G., 1986. Large deformations under vertical edge loads of annular membranes with various strain energy densities. J. NonLinear Mech. 21, 257-267.

Gomez-Navarro, C., Burghard, M., Kern, K., 2008. Elastic properties of chemically derived single graphene sheets. Nano Lett. 8, 2045-2049.

Jin, C., Wang, X.D., 2008. A theoretical study of a thin film delamination using shaft-loaded blister test: Constitutive relation without delamination. J. Mech. Phys. Solids 56, 2815-2831.

Jin, C., Li, J., Wang, Y., 2016. Spherical indentation testing of separator membranes for lithium-ion batteries. Journal of Power Sources, to be submitted.

Ju, B.F., Liu, K.K., Ling, S.F., Ng, W.H., 2002. A novel technique for characterizing elastic properties of thin biological membrane. Mech. Mater. 34, 749-754. 
Ju, B.F., Wan, K.T., Liu, K.K., 2004. Indentation of a square elastomeric thin film by a flat-ended cylindrical punch in the presence of long-range intersurface forces. J. Appl. Phys. 96, 6159-6163.

Haque, M.A., Saif, M.T.A., 2002. Application of MEMS force sensors for in situ mechanical characterization of nano-scale thin films in SEM and TEM. Sens. Actuators A 97-98, 239-245.

Hencky, H., 1915. Über den Spannungszustand in kreisunden Platten. Z. Math. Phys. 63, 311-317.

Kang, S.H., Fang, T.H., Hong, Z.H., 2013. Electrical and mechanical properties of graphene oxide on flexible substrate. Journal of Physics and Chemistry of Solids 74, 1783-1793.

Komaragiri, U., Begley, M.R., Simmonds, J.G., 2005. The mechanical response of freestanding circular elastic films under point and pressure loads. ASME J. Appl. Mec. 72, 203-212.

Lee, C., Wei, X., Kysar, J.W., Hone, J., 2008. Measurement of the elastic properties and intrinsic strength of monolayer graphene. Science 321, 3858.

Leseman, Z.C., Mackin, T.J., 2007. Indentation testing of axisymmetric freestanding nanofilms using a MEMS load cell. Sens. Actuators A 134, 264270 .

Liu, K.K., Ju, B.B., 2001. A novel technique for mechanical characterization of thin elastomeric membrane. J. Phys. D: Appl. Phys. 34, L91-L94.

Nadler, B., Tang, T., 2008. Decohesion of a rigid punch from nonlinear membrane undergoing finite axisymmetric deformation. Int. J. Non-linear Mech. 43, 716-721.

Poilane, C., Delobell, P., Lexcellent, C., Hayashi, S., Tobushi, H., 2000. Analysis of the mechanical behavior of shape memory polymer membranes by nanoindentation, bulging and point membrane delection tests. Thin Solid Films 379, 156-165.

Poot, M., van der Zant, H.S.T., 2008. Nanomechanical properties of few-layer graphene membranes. Appl. Phys. Lett. 92, 063111. 
Rausch, M.K., Kuhl, E., 2013. On the effect of prestrain and residual stress in thin biological membranes. Journal of the Mechanics and Physics of Solids 61, 1955-1969.

Schwerin, E., 1929. Über Spannungen und Formänderungen Kreisringförmiger Membranen. Z. techn. Phys. 12, 651-659.

Scott, O.N., Begley, M.R., Komaragini, U., Mackin, T.J., 2004. Indentation of freestanding circular elastomer films using spherical indenters. Acta Materialia 52, 4877-4885.

Small, M.K., Nix, W.D., 1990. Analysis of the accuracy of the bulge test in determining the mechanical properties of thin films. J. Mat. Res. 7, 1553-1563.

Steigmann, D.J., 1986. Proof of a conjecture in elastic membrane theory. J. Appl. Mech. 53, 955-956.

Vlassak, J.J., Nix, W.D., 1992. A new bulge test technique for the determination of Young's modulus and Poisson's ratio of thin films. J. Mat. Res. 7, 3242-3249.

Yang, W.H., Feng, W.W., 1970. On axisymmetrical deformations of nonlinear membranes. J. Appl. Mech. 34, 1002-1011.

Yang, W.H., Hsu, K.H., 1971. Indentation of a circular membrane. J. Appl. Mech. 36, 227-230.

Zheng, D.W., Xu, Y.H, Tsai, P., Tu, K.N., Patterson, P., Zhao, B., Lui, Q.Z., Brongo, M., 2000. Mechanical property measurement of thin polymeric low-dielectric constant films using bulge testing method. Appl. Phys. Lett. 76, 2008-2010. 
Table 1: Summary of Dimensional and Dimensionless Parameters Used in Derivation

\begin{tabular}{|c|c|}
\hline Dimensional parameters & Dimensionless parameters \\
\hline Radial position: $\mathrm{r}(\mathrm{m})$ & $y=r^{2} / a^{2}$ \\
\hline Radius of spherical indenter: $\mathrm{R}(\mathrm{m})$ & $\lambda=h R / a^{2}$ \\
\hline Radius of contact circle: $c(\mathrm{~m})$ & $\zeta=c^{2} / a^{2}$ \\
\hline Gradient of profile: $d w / d r$ & $\phi=(r / h)(d w / d r)$ \\
\hline External load: $P_{0}(\mathrm{~N})$ & $F=4 a^{2} P_{0} /\left(\pi E h^{4}\right)$ \\
\hline Radial membrane stress: $N_{r}(\mathrm{~N} / \mathrm{m})$ & $S=8 r^{2} N_{r} /\left(E h^{3}\right)$ \\
\hline Residual membrane stress: $N_{0}(\mathrm{~N} / \mathrm{m})$ & $n_{0}=8 a^{2} N_{0} /\left(E h^{3}\right)$ \\
\hline Ratio: $N_{0}\left[4 \pi^{2} a^{2} /\left(E h P_{0}^{2}\right)\right]^{1 / 3}$ & $\xi=n_{0} /\left(2 F^{2 / 3}\right)$ \\
\hline
\end{tabular}

Table 2: Summary of the Exact Solution of Spherical Indentation Problem in Parametric Form

\begin{tabular}{|c|c|c|}
\hline & Problem with Boundary Condition (a) & Problem with Boundary Condition (b) \\
\hline$C_{1}>0$ & $\begin{array}{c}0 \xi^{3 / 2}<\xi^{*} \\
\text { obtain } \theta_{m} \text { and } \theta_{n} \text { from Eq. (75) } \\
\text { obtain } \zeta, \epsilon_{m} \text { and } d \text { from Eqs. (79)-(81) }\end{array}$ & $\begin{array}{c}0<\nu<1-\nu^{*} \\
\text { obtain } \theta_{m} \text { and } \theta_{n} \text { from Eq. (77) } \\
\text { obtain } \zeta, \epsilon_{m} \text { and } d \text { from Eqs. (79)-(81) }\end{array}$ \\
\hline$C_{1}=0$ & $\begin{array}{c}\xi^{3 / 2}=\xi^{*} \\
\text { obtain } \zeta, \epsilon_{m} \text { and } d \text { from Eqs. (84)-(86) }\end{array}$ & $\begin{array}{c}\nu+\nu^{*} \\
\text { obtain } \zeta, \epsilon_{m} \text { and } d \text { from Eqs. (84)-(86) }\end{array}$ \\
\hline$C_{1}<0$ & $\begin{array}{c}\xi^{3 / 2}>\xi^{*} \\
\text { obtain } \theta_{m} \text { and } \theta_{n} \text { from Eq. (76) } \\
\text { obtain } \zeta, \epsilon_{m} \text { and } d \text { from Eqs. (81)-(83) }\end{array}$ & $\begin{array}{c}\text { obtain } \theta_{m} \text { and } \theta_{n} \text { from Eq. (78) } \\
\text { obtain } \zeta, \epsilon_{m} \text { and } d \text { from Eqs. (81)-(83) }\end{array}$ \\
\hline
\end{tabular}


Table 3: Summary of Point-Load Solution in Parametric Form

\begin{tabular}{|c|c|c|}
\hline & Problem with Boundary Condition (a) & Problem with Boundary Condition (b) \\
\hline$C_{1}>0$ & $\begin{array}{c}0<\xi^{3 / 2}<3 / 4 \\
\text { obtain central deflection from Eq. (87) }\end{array}$ & $\begin{array}{c}0<\nu<1-\nu^{* *} \\
\text { obtain central deflection from Eq. (89) }\end{array}$ \\
\hline$C_{1}=0$ & $\begin{array}{c}\xi^{3 / 2}=3 / 4 \\
\text { obtain central deflection from Eq. (91) }\end{array}$ & $\begin{array}{c}\qquad \nu=1-\nu^{* *} \\
\text { obtain central deflection from Eq. (91) }\end{array}$ \\
\hline$C_{1}<0$ & $\begin{array}{c}\xi^{3 / 2}>3 / 4 \\
\text { obtain central deflection from Eq. (88) }\end{array}$ & $\begin{array}{c}1-\nu^{* *}<\nu<0.5 \\
\text { obtain central deflection from Eq. (90) }\end{array}$ \\
\hline
\end{tabular}

Table 4: Quantitative Comparison among Experimental Data, Approximate Solution, and Exact Solution

\begin{tabular}{|c|c|c|c|}
\hline & \multicolumn{3}{|c|}{$\mathrm{d}=2.00 \mathrm{~mm}$} \\
\hline & Experimental Data (Mean) & Approximate Solution & Exact Solution \\
\hline$R / a=0.03$ & $0.0030 \mathrm{~N}$ & $0.0013 \mathrm{~N} \quad 56.7 \%$ difference & $0.0023 \mathrm{~N} 23.3 \%$ difference \\
\hline$R / a=0.14$ & $0.0038 \mathrm{~N}$ & $0.0020 \mathrm{~N} 47.4 \%$ difference & $0.0026 \mathrm{~N} 31.6 \%$ difference \\
\hline$R / a=0.50$ & $0.0049 \mathrm{~N}$ & $0.0027 \mathrm{~N} 44.9 \%$ difference & $0.0031 \mathrm{~N} 36.7 \%$ difference \\
\hline \multirow[t]{3}{*}{$R / a=1.00$} & $0.0050 \mathrm{~N}$ & $0.0031 \mathrm{~N} 38.0 \%$ difference & $0.0036 \mathrm{~N} 28.0 \%$ difference \\
\hline & \multicolumn{3}{|c|}{$\mathrm{d}=4.00 \mathrm{~mm}$} \\
\hline & Experimental Data (Mean) & Approximate Solution & Exact Solution \\
\hline$R / a=0.03$ & $0.0196 \mathrm{~N}$ & $0.0106 \mathrm{~N} 45.9 \%$ difference & $0.0197 \mathrm{~N} \quad 0.51 \%$ difference \\
\hline$R / a=0.14$ & $0.0226 \mathrm{~N}$ & $0.0156 \mathrm{~N} 31.0 \%$ difference & $0.0225 \mathrm{~N} \quad 0.44 \%$ difference \\
\hline$R / a=0.50$ & $0.0322 \mathrm{~N}$ & $0.0224 \mathrm{~N} 30.4 \%$ difference & $0.0293 \mathrm{~N} 9.01 \%$ difference \\
\hline$R / a=1.00$ & $0.0391 \mathrm{~N}$ & $0.0263 \mathrm{~N} 32.7 \%$ difference & $0.0384 \mathrm{~N} 1.79 \%$ difference \\
\hline
\end{tabular}




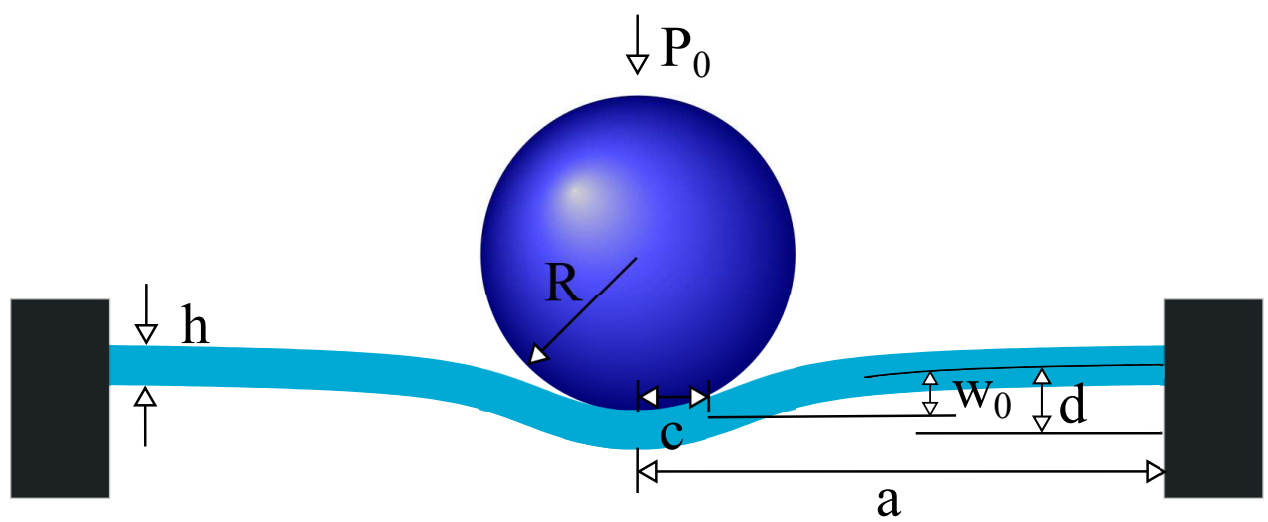

Figure 1: Schematic diagram (not drawn to scale) of the spherical indentation test for freestanding films. 


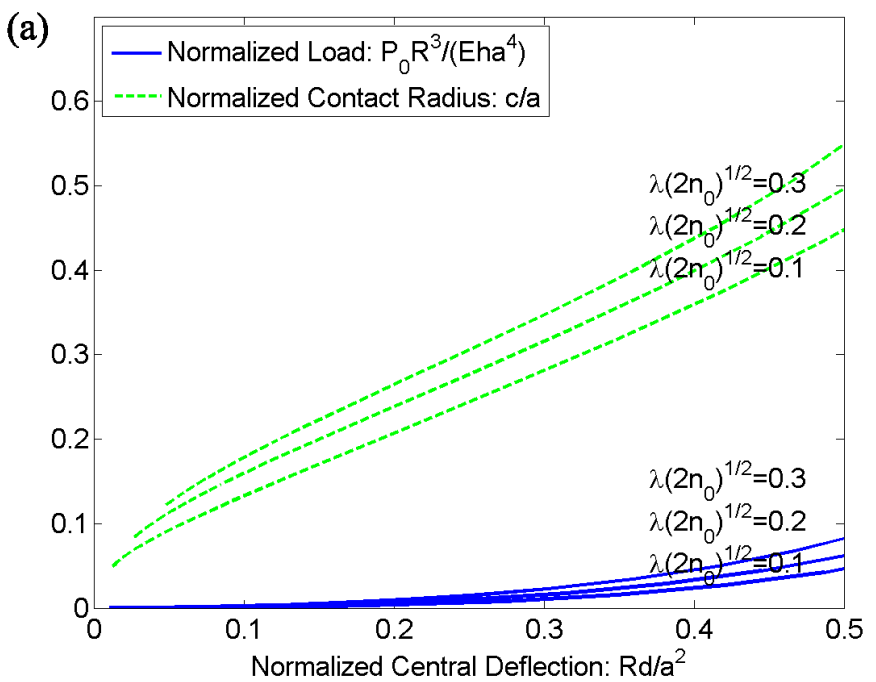

(b)

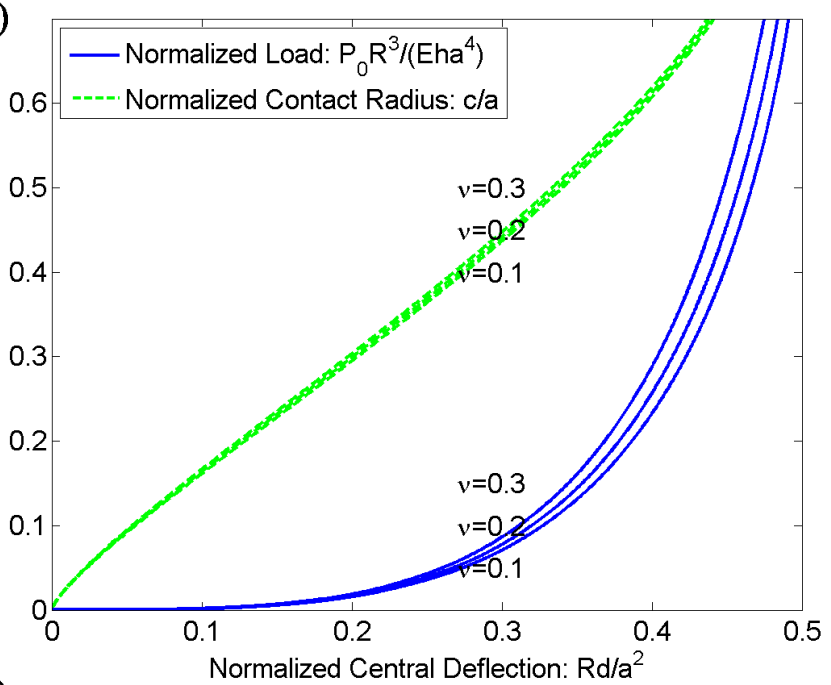

(c)

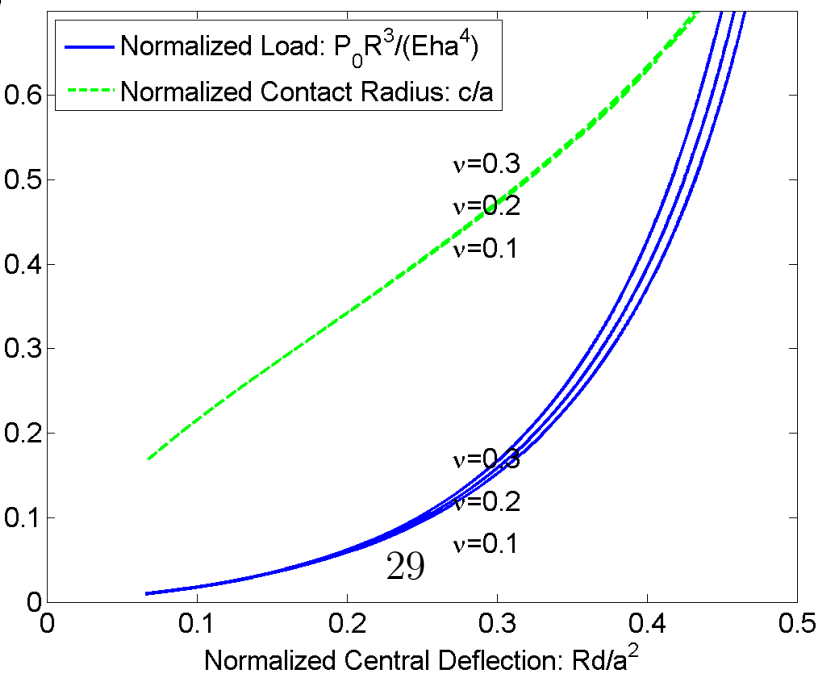

Figure 2: For the spherical indentation problem with boundary condition (a), the relationship among load, central deflection, and contact radius has been plotted in (a). For the problem with boundary condition (b), in the limit of zero prestrain, the relationship among load, central deflection, and contact radius has been plotted in (b). For the problem with boundary condition (b), when $\lambda\left(2 n_{0}\right)^{1 / 2}=0.9$, the relationship among load, central deflection, and contact radius has been plotted in (c). 


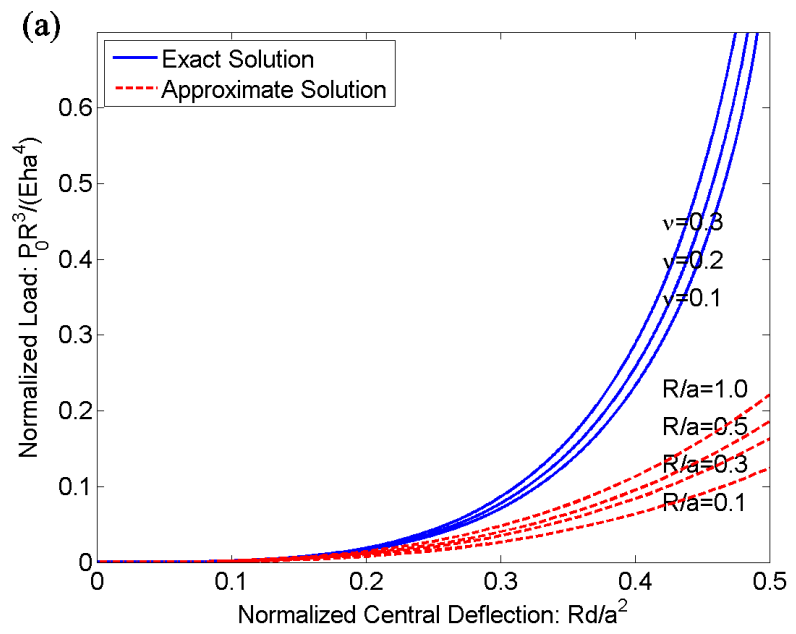

(b)

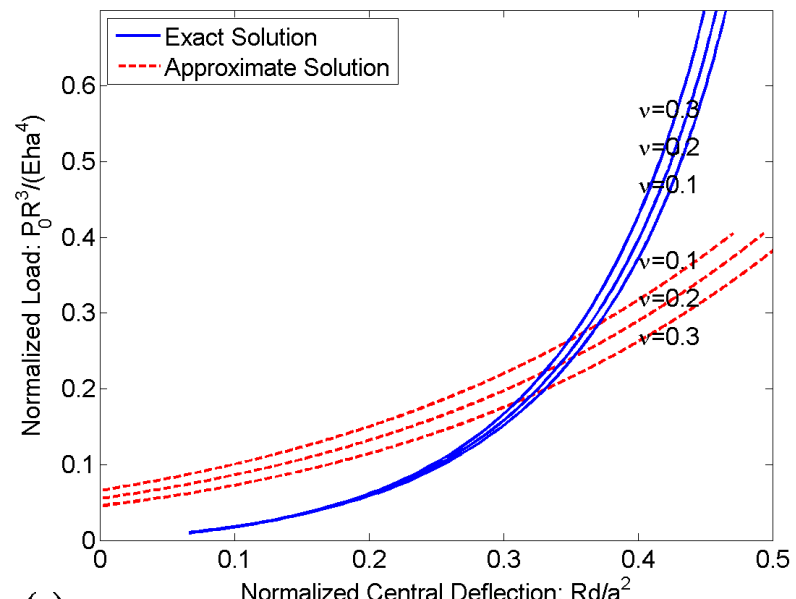

(c)

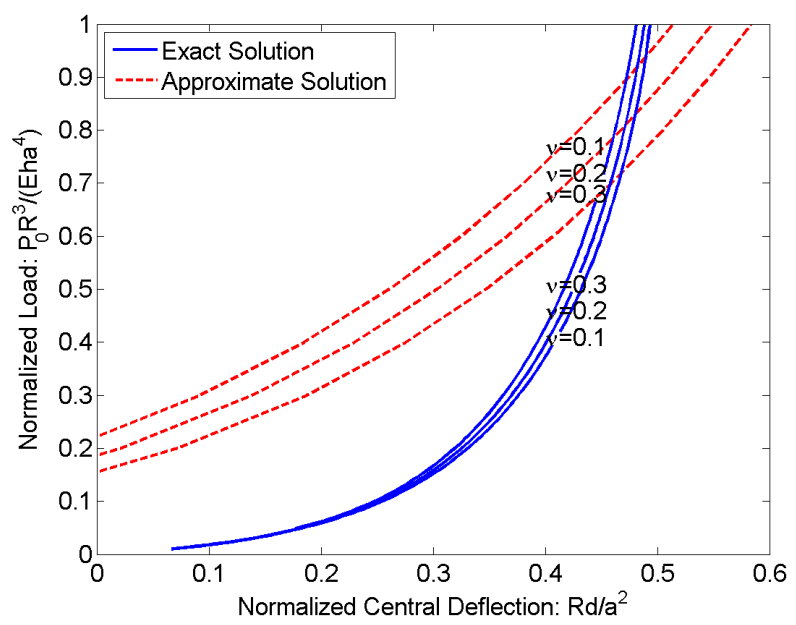

30

Figure 3: For the problem with boundary condition (b), in the limit of zero prestrain, the load-deflection curves based on the approximate solution and the exact solution have been compared in (a). For the problem with boundary condition (b), when $\lambda\left(2 n_{0}\right)^{1 / 2}=0.9$ and $R / a=0.3$, the relationship among load, central deflection, and contact radius has been plotted in (b).For the problem with boundary condition (b), when $\lambda\left(2 n_{0}\right)^{1 / 2}=0.9$ and $R / a=1.0$, the relationship among load, central deflection, and contact radius has been plotted in (c). 

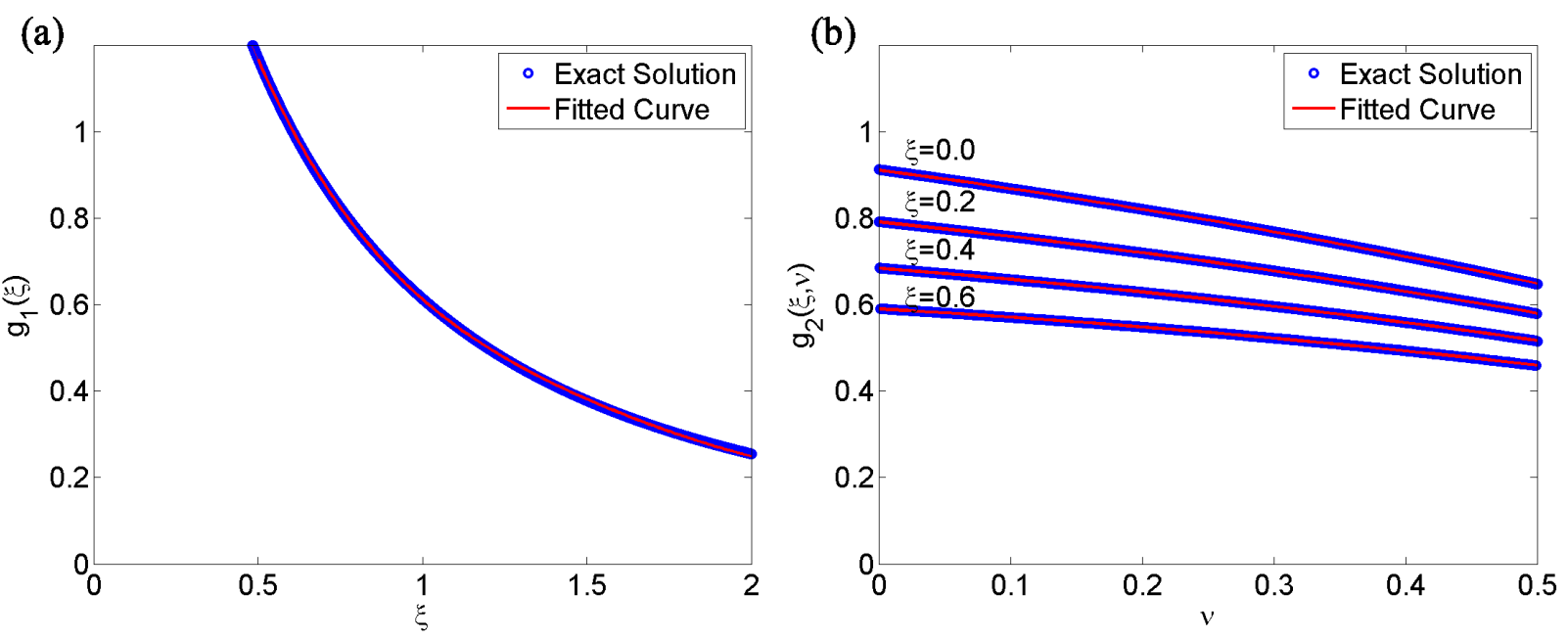

Figure 4: (a) The plot of $g_{1}(\xi)$ as a function of $\xi$. The fitted curve shows $g_{1}(\xi) \approx 0.1782 \xi^{4}-$ $1.191 \xi^{3}+3.106 \xi^{2}-4.018 \xi+2.537$. (b) The plot of $g_{2}(\xi, \nu)$ as a function of $\nu$ when $\xi=0.0$, $0.2,0.4$, and 0.6. The fitted curves show $\left.g_{2}(\nu)\right|_{\xi=0.0} \approx-0.2499 \nu^{2}-0.3999 \nu+0.9109$, $\left.g_{2}(\nu)\right|_{\xi=0.2} \approx-0.2161 \nu^{2}-0.3136 \nu+0.791,\left.g_{2}(\nu)\right|_{\xi=0.4} \approx-0.1962 \nu^{2}-0.2357 \nu+0.6838$, and $\left.g_{2}(\nu)\right|_{\xi=0.6} \approx-0.1739 \nu^{2}-0.1733 \nu+0.5897$. 


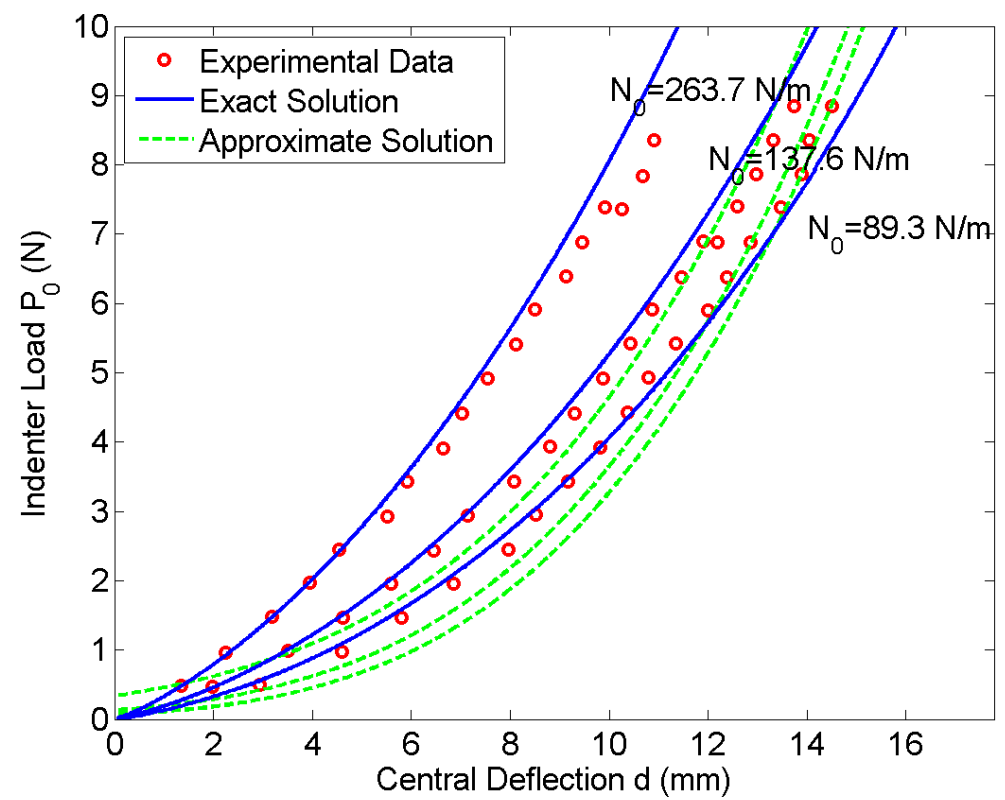

Figure 5: The comparison of the analytical solutions with the experimental data provided by Bhatia and Nachbar (1968). 

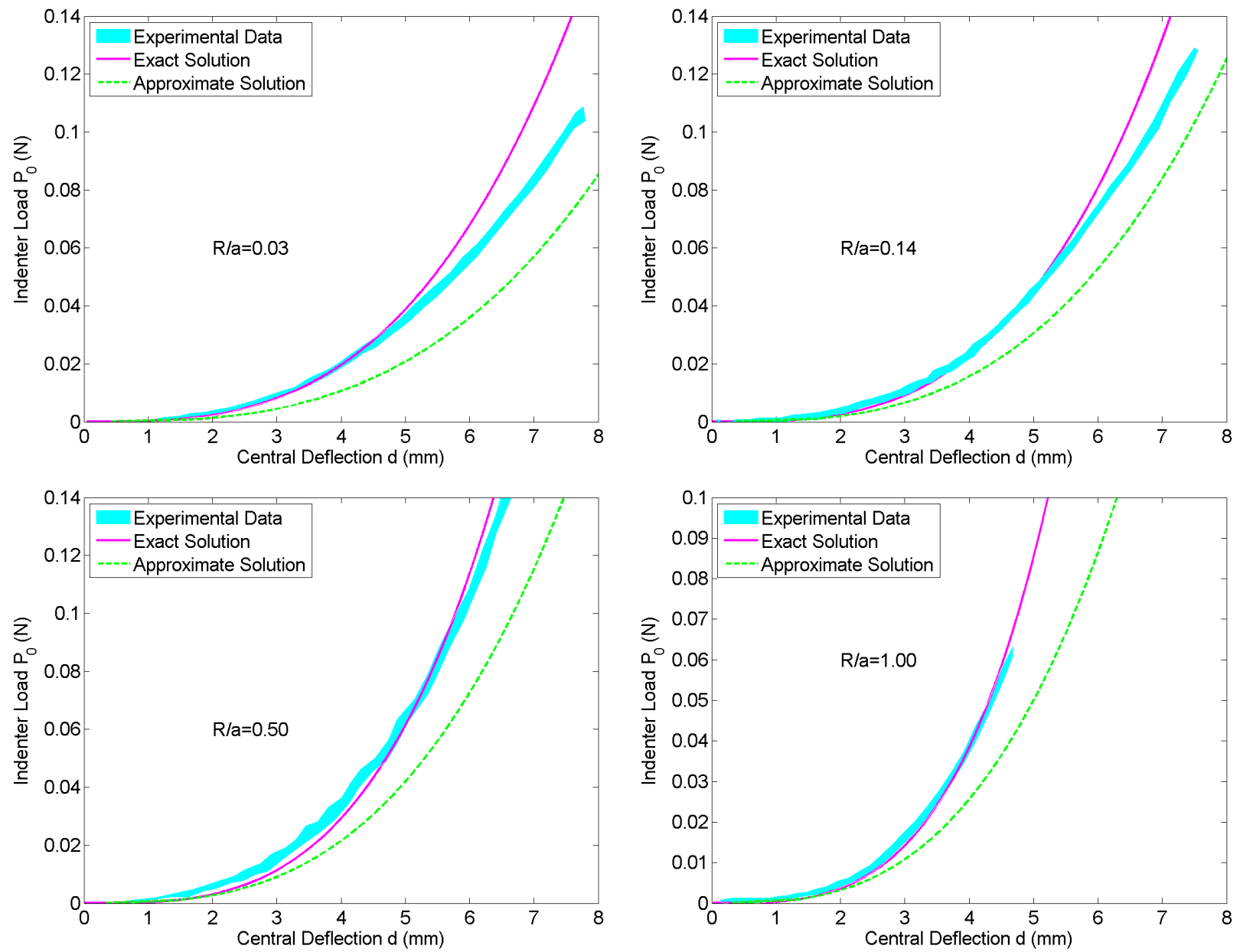

Figure 6: The comparison of the analytical solutions with the experimental data provided by Scott et al. (2004). 

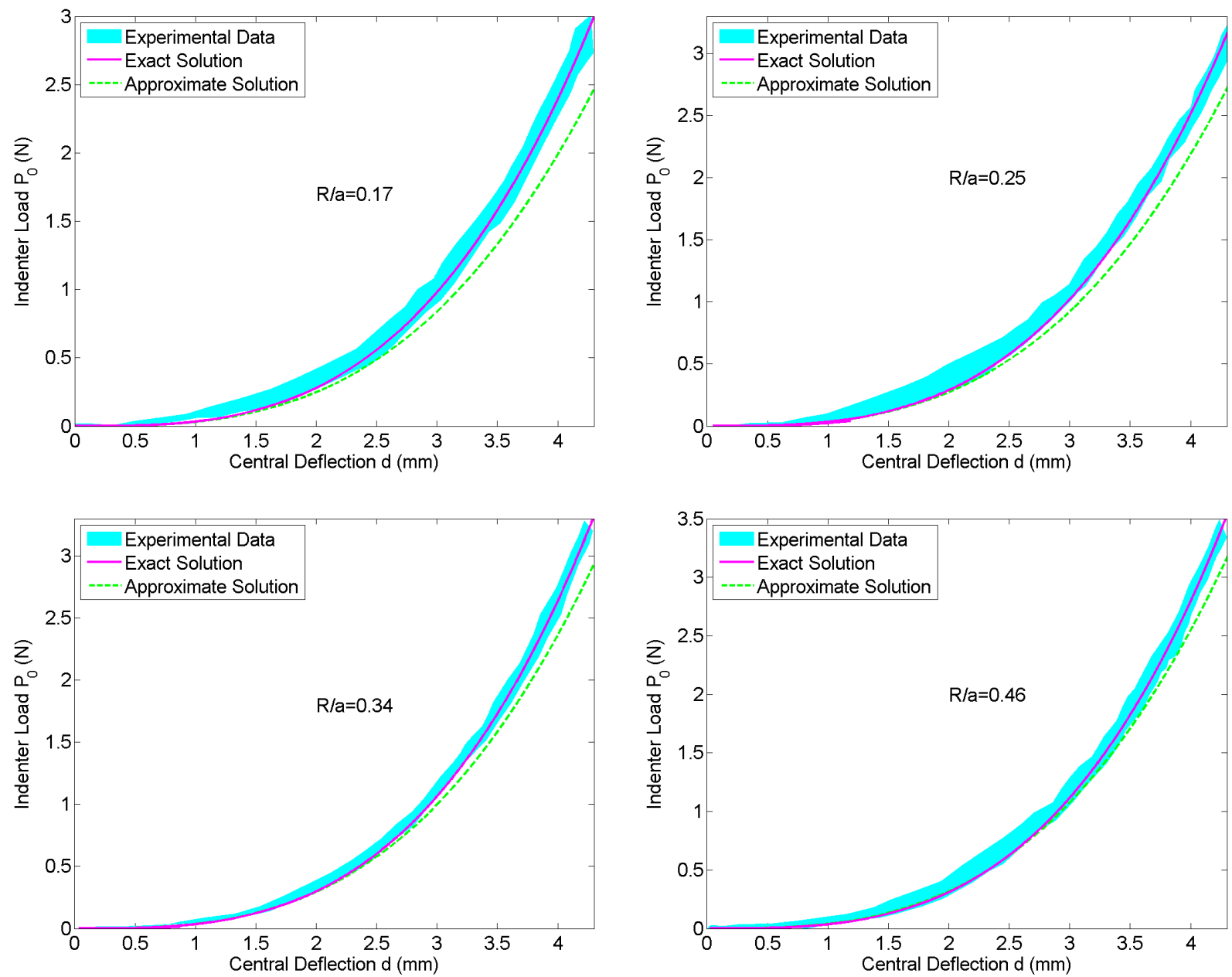

Figure 7: The comparison of the analytical solutions with the experimental data. 